\title{
Mad2 is required for inhibiting securin and cyclin B degradation following spindle depolymerisation in meiosis I mouse oocytes
}

\author{
Hayden A Homer ${ }^{1,2}$, Alex McDougall $^{2}$, Mark Levasseur ${ }^{2}$, Alison P Murdoch ${ }^{1,3}$ \\ and Mary Herbert ${ }^{1,3^{\prime}}$ \\ ${ }^{1}$ Newcastle Fertility Centre at Life, International Centre for Life, Times Square, Newcastle upon Tyne, NE1 4EP, \\ UK, ${ }^{2}$ School of Cell and Molecular Biosciences, The Medical School, Framlington Place, University of Newcastle, \\ Newcastle upon Tyne, NE2 4HH, UK and ${ }^{3}$ School of Surgical and Reproductive Sciences, The Medical School, \\ Framlington Place, University of Newcastle, Newcastle upon Tyne, NE2 4HH, UK
}

Correspondence should be addressed to HA Homer or M Herbert, Newcastle Fertility Centre at Life, International Centre for Life, Times Square, Newcastle upon Tyne, NE1 4EP, UK; Email: mary.herbert@ncl.ac.uk or h.a.homer@newcastle.ac.uk

A McDougall is now at UMR 7009 CNRS/Université Pierre et Marie Curie (Paris VI), Observetoire Océanologique, 06230 Villefranche-sur-Mer, France

\begin{abstract}
Mad2 is a pivotal component of the spindle assembly checkpoint (SAC) which inhibits anaphase promoting complex/cyclosome (APC/C) activity by sequestering Cdc20 thereby regulating the destruction of securin and cyclin B. During mitosis, spindle depolymerisation induces a robust Mad2-dependent arrest due to inhibition of securin and cyclin B destruction. In contrast to mitosis, the molecular details underpinning the meiosis I arrest experienced by mouse oocytes exposed to spindle depolymerisation remain incompletely characterised. Notably, the role of Mad2 and the fate of the anaphase-marker, securin, are unexplored. As shown previously, we find that spindle depolymerisation by nocodazole inhibits first polar body extrusion (PBE) and stabilises cyclin B and cyclin-dependent kinase 1 activity in mouse oocytes. Here we show that stabilisation of cyclin B in nocodazole can be sustained for several hours and is associated with stabilisation of securin. These effects are SAC-mediated as, in oocytes depleted of the majority of Mad2 by morpholino antisense, securin and cyclin B are destabilised and $15 \%$ of oocytes undergo PBE. This reflects premature APC/C activation as a mutant form of cyclin B lacking its APC/C degradation signal is stable in Mad2-depleted oocytes. Moreover, homologues do not disjoin during the prolonged meiosis I arrest $(>18 \mathrm{~h})$ induced by nocodaozole indicating that a non-cleavage mechanism is insufficient on its own for resolution of arm cohesion in mammalian oocytes. In conclusion, when all kinetochores lack attachment and tension, mouse oocytes mount a robust Mad2-dependent meiosis I arrest which inhibits the destruction of securin and cyclin B.
\end{abstract}

Reproduction (2005) 130 829-843

\section{Introduction}

The spindle assembly checkpoint (SAC) represents a highly conserved network comprised primarily of the Mad (mitotic arrest-deficient) and Bub (budding uninhibited by benzimidazole) proteins (Musacchio \& Hardwick 2002, Taylor et al. 2004). During mitosis, the SAC averts chromosome missegregation by coupling anaphase onset and mitotic exit to the establishment of stable kinetochore-microtubule linkages. In the presence of improperly attached kinetochores, Mad2 and BubR1 sequester Cdc20, the substrate-targeting subunit of a multimeric ubiquitin ligase called the anaphase-promoting complex/cyclosome (APC/C). Once sister kinetochores attain stable bipolar attachments, the SAC is disabled and
$\mathrm{APC} / \mathrm{C}^{\mathrm{Cdc} 2 \mathrm{O}}$-mediated ubiquitination of securin and cyclin B earmarks these proteins for proteolysis by the $26 \mathrm{~S}$ proteasome (Castro et al. 2005). Securin destruction liberates the endopeptidase separase to cleave cohesin, a molecular glue responsible for tethering sister chromatids, whilst cyclin B destruction leads to inactivation of cyclin-dependent kinase 1 (Cdk1) culminating in anaphase and mitotic exit respectively (Uhlmann 2003, Castro et al. 2005).

Following $S$ phase in somatic cells, cohesins unite replicated sister chromatids along both arms and centromeres (Uhlmann 2003). During vertebrate mitosis however, the bulk of arm cohesins dissociate during prometaphase leaving primarily centromeric cohesins and small amounts of arm cohesins to be resolved by separase at anaphase (Uhlmann 2003, Giménez-Abián et al. 2004). Small 
residual levels of arm cohesins are capable of maintaining arm cohesion up until anaphase onset in unperturbed cells but can be induced to dissociate completely in cells in which prometaphase is sufficiently delayed by spindle poisons (Giménez-Abián et al. 2004). Given that spindle poisons activate the SAC (Li \& Benezra 1996, Dobles et al. 2000, Skoufias et al. 2001, Meraldi et al. 2004, Michel et al. 2004), the corollary of this is that during mitosis, arm cohesion can be resolved in the face of an active SAC.

Meiosis is a specialized cell division involving two rounds of chromosome segregation (meiosis I and meiosis II) without an intervening round of DNA replication, resulting in halving of the DNA content. In mammalian oocytes, meiosis is a discontinuous multi-stage process. Following recombination, mammalian oocytes undergo a prophase I arrest identifiable by the presence of a germinal vesicle (GV). Resumption and completion of meiosis I are marked by GV breakdown (GVBD) and first polar body extrusion (PBE) respectively following which oocytes arrest at metaphase II (Fig. 1A). Exit from meiosis I is brought about by inactivation of Cdk1 through degradation of its cyclin B regulatory subunit (Hampl \& Eppig
1995, Winston 1997). Unlike mitosis and meiosis II in which sister chromatids separate, during anaphase I, pairs of recombined homologues segregate or 'disjoin'. Given that recombination produces homologues that are united beyond chiasmata by arm cohesion, the latter must be resolved for homologue disjunction to occur (Watanabe 2004).

Although spindle poisons have been utilized on numerous occasions to delay or arrest meiosis I in mouse oocytes, the molecular details underpinning these responses remain incompletely characterised. In mitotic cells, spindle depolymerisation induces a robust Mad2dependent cell-cycle arrest secondary to APC/C ${ }^{\mathrm{Cdc} 20}$ inhibition and stabilisation of securin and cyclin B (Li \& Benezra 1996, Dobles et al. 2000, Meraldi et al. 2004, Michel et al. 2004, Morrow et al. 2005). In mouse oocytes, both short-term (3-6h) and long-term (14-16 h) spindle depolymerisation induces a meiosis I arrest (Soewarto et al. 1995, Brunet et al. 1999, Lefebvre et al. 2002, Brunet et al. 2003). Although mouse oocytes undergo a transient Mad2-dependent delay in meiosis I when subjected to subtle kinetochore-microtubule defects (Wassmann et al.
A

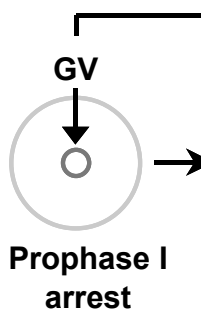
arrest

\section{Meiosis I} GVBD

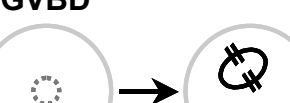

Metaphase I

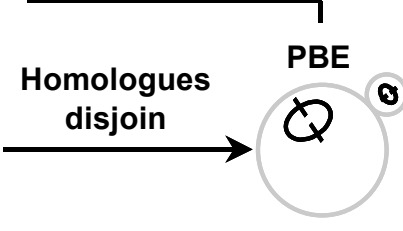

Metaphase II
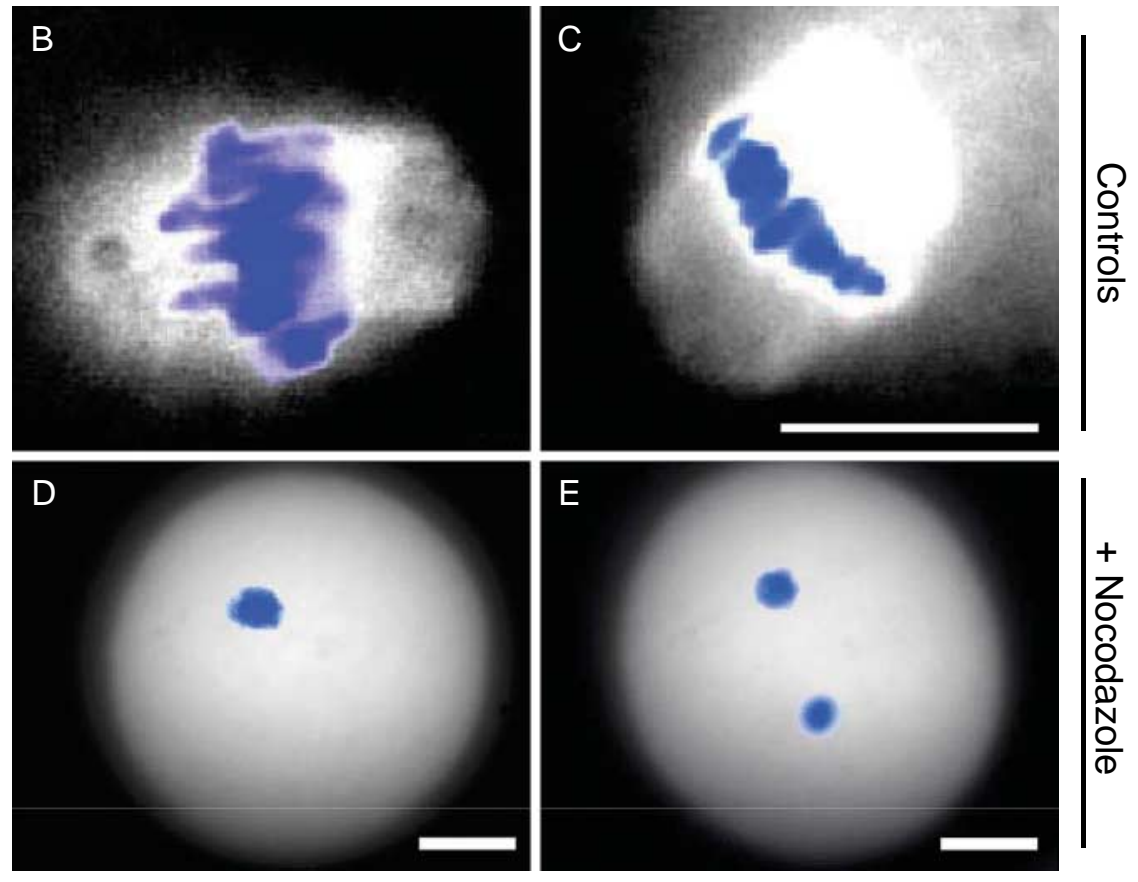

Figure 1 (A) Schematic representation of meiosis I. See main text for further details. (B to E) The spindle is depolymerised by $5 \mu \mathrm{M}$ nocodazole. Oocytes were fixed and immunostained for microtubules (white) using a mouse anti- $\beta$-tubulin as the first layer followed by FITC-conjugated anti-mouse as the second layer. DNA was stained using propidium iodide (blue). (B and C) Control oocytes fixed at $4 \mathrm{~h}$ post-GVBD (B) and at $5 \mathrm{~h}$ post-GVBD (C). (D and E) Oocytes cultured in nocodazole-treated medium and fixed at $18-20 \mathrm{~h}$ post-GVBD. Note that most nocodazole-treated oocytes displayed a single group of DNA (D) whilst $\sim 25 \%$ displayed two or more well defined groups (E). Oocytes fixed after shorter incubation periods in nocodazole ( $4-5 \mathrm{~h}$ post-GVBD) also revealed the absence of a spindle (data not shown). Scale bars $=20 \mu \mathrm{m}$. 
2003), it is not known whether the meiosis I arrest in response to complete spindle depolymerisation is mediated by Mad2. Moreover, whilst securin, one of the principal downstream targets of the SAC, has previously been examined in unperturbed meiosis I mouse oocytes (Herbert et al. 2003, Terret et al. 2003, Tsurumi et al. 2004, Homer et al. 2005a), the fate of securin following exposure to spindle poisons is unknown.

Here we examine mouse oocytes during prolonged exposure to nocodazole at doses which depolymerise the spindle completely. We utilise morpholino-induced depletion of Mad2 to disable the SAC and timelapse imaging of GFP-tagged chimerae to facilitate real-time analysis of securin and cyclin B (Ledan et al. 2001, Herbert et al. 2003, Tsurumi et al. 2004, Homer et al. 2005a). Consistent with previous reports, spindle depolymerisation prevents PBE, stabilises Cdk1 and cyclin B, while inhibiting homologue disjunction (Soewarto et al. 1995, Brunet et al. 1999, Lefebvre et al. 2002, Brunet et al. 2003). Here we expand on this data by showing that inhibition of cyclin B destruction following spindle depolymerisation can be maintained for several hours and is accompanied by stabilisation of securin. Meiosis I arrest requires Mad2 - as securin and cyclin B are rendered unstable $-15 \%$ of oocytes undergo PBE following Mad2 depletion. Moreover, protein destruction is mediated by unrestrained APC/C activity as a cyclin B construct lacking its APC/C degradation signal is stable in Mad2 depleted oocytes cultured in nocodazole. Furthermore, given that during a prolonged meiosis I arrest homologue disjunction does not occur, another important implication of our findings is that arm cohesion cannot be completely resolved by a non-cleavage mechanism in mammalian oocytes as it can in mitotic cells. Our data therefore demonstrate that mouse oocytes are capable of mounting a robust SAC response to spindle depolymerisation which, like mitosis, targets securin and cyclin B. However, unlike mitosis in which resolution of arm cohesion can be resolved in the face of an active SAC (Giménez-Abián et al. 2004), during female mammalian meiosis I, conditions which inhibit the SAC also inhibit the resolution of arm cohesion.

\section{Materials and Methods}

\section{Collection, culture and drug treatment of oocytes}

Ovaries were isolated from 4-6 week-old MF1 female mice 46-48 h after treatment with 7.5-10 international units of pregnant mares serum gonadotrophin (PMSG). Ovaries were placed in a Petri dish with pre-warmed $\left(37^{\circ} \mathrm{C}\right) \mathrm{M} 2$ medium (Sigma) supplemented with $50 \mu \mathrm{g} / \mathrm{ml}$ dibutyryl cyclic AMP (dbcAMP) which prevents oocytes from undergoing GVBD. Cumulus-free, fully grown, GVintact oocytes were released by puncturing antral follicles with a fine needle on the stage of a dissecting microscope. For in vitro maturation, oocytes with a clearly visible GV were transferred to droplets of M16 medium (Sigma) under mineral oil (Sigma) at $37^{\circ} \mathrm{C}$ in a humidified atmosphere of $5 \% \mathrm{CO}_{2}$ in air. For experiments in which oocytes were treated with nocodazole, $5 \mathrm{mg} / \mathrm{ml}$ nocodazole (Sigma) in DMSO stock was diluted into M16 medium to a final concentration of $5 \mu \mathrm{M}$.

\section{Microinjection}

GV-stage oocytes were microinjected in droplets of prewarmed $\left(37^{\circ} \mathrm{C}\right)$ dbcAMP-supplemented M2 medium under mineral oil. Holding and injection pipettes were made from sterile filament-free GC100T-10 glass (Clarke Electromedical Instruments, Reading, Berkshire, UK) using a P-97 micropipette puller (Sutter Instrument Co., Novato, CA, USA) following which they were given an angle of approximately $50^{\circ}$. Microinjections were carried out on a Nikon Diaphot ECLIPSE TE 300 (Nikon UK Ltd, Kingston upon Thames Surrey, UK) inverted microscope equipped with Narishige MM0-202N hydraulic three-dimensional micromanipulators (Narishige Inc., Sea Cliff, NY, USA) using a $10 \times / 0.25$ n.a. objective combined with a $2.5 \times$ magnifier. About $1 \mu$ l of test solutions was micropipetted into capillary tubes and sandwiched between oil to reduce evaporation and loaded tubes were mounted onto glass slides using paraffin wax. Injection micropipettes were introduced across the oil layer into the solution and tip-filled by reducing the balance pressure of the microinjector. After filling, the meniscus was stabilised by adjusting the balance pressure. GV-stage oocytes were immobilized using a holding pipette and the tip of the injection pipette was introduced across the zona pellucida and oolemma into the ooplasm. A pressure pulse was applied (4 to10 psi) using a semi-automatic Narishige IM 300 microinjection device (Narishige Inc.) to microinject test solutions in amounts varying from $1-5 \%$ of the oocyte volume depending on what was being injected. Needle and/or final concentrations of test solutions microinjected are indicated below. Following microinjection, oocytes were transferred to fresh dbcAMP-supplemented M2 medium and allowed to recover for at least $30 \mathrm{~min}$ prior to longer term culture in M16 medium, in a humidified atmosphere of $5 \% \mathrm{CO}_{2}$ in air at $37^{\circ} \mathrm{C}$.

\section{GFP constructs, morpholinos and GFP antisense}

To construct a human Mad2-green fluorescent protein complex (hMad2-GFP), full-length sequence coding for hMad2 was subcloned into the pMDL2 transcription vector, a derivative of pRN3 now containing the sequence coding for mmGFP (Levasseur \& McDougall 2000, Homer et al. 2005a, Homer et al. 2005b). Capped mRNA consisting of hMad2 fused via its C-terminus through a 5-amino acid linker (AGAQF) to the second $\mathrm{N}$-terminal amino acid residue of mmGFP was produced using the T3 mMESSAGE mMACHINE kit (Ambion Inc., Austin, TX, USA) and dissolved in nuclease-free water to a final concentration of $1-1.5 \mu \mathrm{g} / \mu \mathrm{l}$. Constructs consisting of mmGFP linked to 
human cyclin B1 and human securin have been described previously (Herbert et al. 2003, Homer et al. 2005a). All mRNA constructs were microinjected at a needle concentration of $0.5-1 \mu \mathrm{g} / \mu \mathrm{l}$.

Morpholino phosphorodiamidate oligomer sequences were supplied by Gene Tools (Gene Tools LLC, Philomath, OR, USA). Homology searches (BLAST and Celera) were performed to ensure no interaction between the Mad2 targeting morpholino and other sequences within the mouse genome. For microinjection, morpholinos were reconstituted using sterile water as recommended by the manufacturer to produce a needle concentration of $17 \mu \mathrm{g} / \mu \mathrm{l}$ and a final oocyte concentration of $0.8 \mu \mathrm{g} / \mu \mathrm{l}$.

The GFP-targeting phosphorothioate-linked DNA antisense (AS) was described previously (Nixon et al. 2002, Homer et al. 2005a) and injected into GV-stage oocytes at a concentration of $0.5 \mu \mathrm{g} / \mu \mathrm{l}$ as described in figure legends and in the main text.

\section{Immunoblotting}

Oocyte samples were collected in sample buffer containing $\beta$-mercaptoethanol and immediately frozen at $-20^{\circ} \mathrm{C}$. Following thaw, samples were heated to $95^{\circ} \mathrm{C}$ for $5 \mathrm{~min}$ and proteins were resolved by standard SDS-polyacrylamide gel electrophoresis on a $12 \%$ gel and electrically transferred to a hydrophobic polyvinylidene difluoride membrane (Hybond-P; Amersham Biosciences, Little Chalfort, Buckinghamshire, UK). Following transfer, nonspecific binding sites were blocked by incubating membranes for $2 \mathrm{~h}$ in 5\% non-fat milk in TBS $(25 \mathrm{mM}$ Tris, $150 \mathrm{mM} \mathrm{NaCl}, \mathrm{pH} 8.0)$ containing $0.05 \%$ Tween 20 (TBST). Membranes were first probed with either the goat polyclonal antibody (sc-6330; Santa Cruz Biotechnology Inc., Santa Cruz, CA, USA) against Mad2 or the rabbit polyclonal antibody (ab6556; Abcam Ltd, Cambridge, UK) against GFP. Following three 5-minute washes in TBST, incubation with horseradish peroxidase-conjugated secondary antibody and a further three TBST washes and one TBS wash, detection was performed using the ECL Plus chemiluminescence system (Amersham Biosciences) according to the manufacturer's protocol. The same membrane was then directly reprobed with the mouse monoclonal antibody (ab8245; Abcam Ltd) against glyceraldehyde-3-phosphate dehydrogenase (GAPDH) as previously described (Homer et al. 2005a). The anti-Mad2 antibody (sc-6330; Santa Cruz Biotechnology Inc.) reacted with a conserved epitope located within the $\mathrm{N}$-terminal region of hMad2. Similar results were obtained with an anti-Mad2 antibody directed against a peptide mapping to the C-terminal region of hMad2 (sc-6329; Santa Cruz Biotechnology, Inc.). The GAPDH signal acted as internal controls to ensure even sample loading and gel transfer. Protein expression was examined by analysing the optical density of the bands obtained in each Western blot analysis using TINA software (Tine Is No Acronym image analysis software environment; www.tina-vision.net).

\section{Timelapse imaging and visualization of DNA in live oocytes}

Imaging was performed using a Nikon Eclipse TE2000-U inverted fluorescence microscope equipped with $20 \times / 0.75$ n.a. and $40 \times / 1.4$ n.a. Plan Fluor oil immersion objectives and a $1.5 \times$ magnification lens, motorized shutters for trans- and epi-fluorescence illumination, motorized excitation and barrier filter wheels, dichroic filter blocks for viewing GFP, DAPI (for Hoechst 33342stained DNA), or rhodamine (for propidium iodide-stained DNA) housed in a rotating turret. A Photometrics CoolSnap $_{\text {нQ }}$ interline cooled charge-coupled device (CCD) camera (Roper Scientific Inc., Trenton, NJ, USA) mounted to the bottom port, a Xenon $150 \mathrm{~W}$ light source (OPTI QUIP, NY, USA) with a Hamamatsu C6979 power supply (Hamamatsu Photonics UK Ltd, Welwyn Garden City, Hertfordshire, UK) and a Prior ProScanll Controller (Prior Scientific, Inc., Cambridge, UK) for automated control of the microscope stage, shutters and filter wheels driven by MetaMorph image processing software (Universal Imaging Corp., Donnington, PA, USA).

Timelapse imaging of oocytes expressing cyclin B-GFP or securin-GFP was performed in stage-fitted dishes containing pre-warmed microdrops of M16 medium under mineral oil maintained at ambient conditions of $5 \% \mathrm{CO}_{2}$ and $37^{\circ} \mathrm{C}$ by means of a modified stage-mounted incubator (Solent, Plymouth, Devon, UK). Two 12-bit images (one brightfield image and one fluorescence image) were collected every 20 min for $14-16 \mathrm{~h}$ using the $20 \times$ objective lens at fixed settings of $1 \times 1$ binning and 100 millisecond exposure times. The dynamics of GFP fluorescence intensities were analysed using MetaMorph software and for each frame of a timelapse series, involved drawing a region of interest over the entire oocyte to measure the average total-oocyte fluorescence intensity which was then background-corrected by subtracting the mean fluorescence value of a cell-free region. The output from this routine was logged in Excel (Microsoft) and plotted against time to generate graphs.

For fluorescence imaging of DNA in live oocytes, oocytes were bathed in Hoechst 33342 (10 $\mu \mathrm{g} / \mathrm{ml}$; Sigma) for $15 \mathrm{~min}$ and imaged using the $20 \times$ objective lens. Images were processed using MetaMorph software and assembled into panels using Adobe Photoshop (Adobe Systems Inc., San Jose, CA, USA).

\section{Immunofluorescence}

Oocytes were fixed and permeabilized for $1 \mathrm{~h}$ in $4 \%$ paraformaldehyde and $0.3 \%$ Triton X-100 in phosphate-buffered saline (PBS). After three 15-minute washes in PBS containing 4\% bovine serum albumin (PBA), oocytes were blocked for $45 \mathrm{~min}$ in $10 \%$ goat serum in PBS. Following an overnight incubation with the mouse monoclonal antibody against $\beta$-tubulin (T-4026; Sigma) in microdrops under oil at $4{ }^{\circ} \mathrm{C}$, oocytes were given three 15-minute washes in PBA followed by a 45-minute incubation in 
FITC-conjugated rabbit anti-mouse IgG (F-5262; Sigma) in microdrops under oil at room temperature. After a further three 15-minute washes in PBA, DNA was stained with propidium iodide $(200 \mu \mathrm{g} / \mathrm{ml})$ in PBS for $20 \mathrm{~min}$. Following another three 15-minute washes in PBA, oocytes were transferred in volumes of approximately $10-20 \mu$ l to polyL-lysine-coated glass slides scored beforehand with a diamond scriber to aid in locating fixed oocytes and mounted in $90 \%$ glycerol in PBS under a cover slip. Images were captured on the fluorescence work station described above using the $40 \times$ objective lens. Images were processed using MetaMorph software and assembled into panels using Adobe Photoshop.

\section{Air-dried chromosome spreads and C-banding}

Chromosome preparations were performed as described previously (Homer et al. 2005a) and essentially involved zona dissolution, hypotonic treatment, methanol/acetic acid fixation and spreading. Chromosome enumeration errors arising from overlapping oocyte spreads were eliminated by individually pipetting oocytes in very small volumes of approximately $1-2 \mu \mathrm{l}$ on to specific positions on poly-L-lysine-coated glass slides which were delineated using a diamond scriber. There was no possibility of chromosomes from PBs affecting chromosome counts as PBs were separated from oocytes at the zona dissolution stage prior to spreading. In each oocyte analysed, chromosomes were analysed under phase-contrast using a Nikon Eclipse E400 inverted microscope equipped with a $100 \times / 1.25$ n.a. Ph3 DL oil immersion lens and a sensitive a Photometrics CoolSnap ${ }_{H Q}$ interline CCD camera (Roper Scientific) mounted to the upper microscope port. Oocytes were excluded from the analysis based on the following criteria: inadequate C-banding for discriminating chromosome morphology; overlapping or clustering of chromosomes or excessive chromosome scatter that precluded an objective assessment of chromosome numbers.

\section{Histone $\mathrm{H} 1$ and myelin basic protein in vitro kinase assays}

Myelin basic protein (MBP) kinase and histone $\mathrm{H} 1$ kinase activities were assayed as previously described (Verlhac et al. 1994, McDougall \& Levasseur 1998). MBP kinase provided a measure of MAP kinase activity and histone $\mathrm{H} 1$ kinase assays provided a measure of $\mathrm{Cdk} 1$ activity. At fixed time points, samples of 6 oocytes were collected after first washing through $17 \mathrm{M}$ glycine three times (this does not alter meiotic progression). The oocytes were then removed in a volume of $2 \mu \mathrm{l}$ and transferred to $8 \mu \mathrm{l}$ reaction buffer ( $25 \mathrm{mM}$ Hepes, $80 \mathrm{mM} \beta$-glycerophosphate, $5 \mathrm{mM}$ EGTA, $10 \mathrm{mM} \mathrm{MgCl}$, $1 \mathrm{mM}$ DDT, $10 \mu \mathrm{g} / \mathrm{ml}$ leupeptin/pepstatin/aprotonin, $0.2 \mathrm{mM}$ AEBSF, $1 \mathrm{mM}$ benzamidine, $100 \mu \mathrm{M} \mathrm{NaVO}$, $5 \mathrm{mM} \mathrm{NaF}, \mathrm{pH} 7.2$ ). At this point the oocytes were snap frozen in liquid nitrogen. After defrosting the samples on ice, which is sufficient to lyse the oocytes, $2 \mu l 6 \times$ reaction mixture was added to the lid of each Eppendorf $(0.9 \mathrm{mg} / \mathrm{ml}$ myelin basic protein (Sigma) or histone $\mathrm{H} 1$ (Sigma type III from calf thymus), $0.6 \mathrm{mM}$ ATP, $0.5 \mathrm{mCi} / \mathrm{ml}$ [32P]ATP, $60 \mu \mathrm{M}$ cAMP-dependent protein kinase inhibitor in $1 \times$ reaction buffer at $\mathrm{pH7.2)}$. The reaction was started synchronously by spinning the Eppendorfs and then transferring them to a water bath at $30^{\circ} \mathrm{C}$ for $30 \mathrm{~min}$. Following this, the reaction was stopped synchronously by adding $2 \times$ sample buffer to the lid of each Eppendorf and spinning in a microfuge. The samples were then heated to $95^{\circ} \mathrm{C}$ for $3 \mathrm{~min}$ and resolved on $15 \%$ polyacrylamide gels. The resolved proteins on the gel were placed in a Phosphorimager (Fuji BAS-1500; Fuji Photo Film Ltd, Bedford, UK) and the incorporation of [32P] measured quantitatively. All experiments were repeated at least three times.

\section{Results}

\section{Spindle depolymerisation stabilises securin and cyclin B for several hours}

Control oocytes of the MF1 strain (MF1 oocytes) assembled a bipolar spindle by $\sim 4-5 \mathrm{~h}$ post-GVBD (Fig. $1 \mathrm{~B}$ and C) and $85 \%$ underwent PBE by $\sim 11 \mathrm{~h}$ postGVBD $(n=115)$ (Homer et al. 2005a). In contrast, in the presence of $5 \mu \mathrm{M}$ nocodazole, the spindle was depolymerised (Fig. 1D and E) and none of the oocytes underwent PBE even up to $18-20 \mathrm{~h}$ post-GVBD $(n=150)$. Thus, unlike the effects of nanomolar concentrations of nocodazole (Wassmann et al. 2003, Shen et al. 2005), the inhibition of PBE induced by spindle depolymerisation can be sustained for prolonged periods consistent with previous reports (Soewarto et al. 1995).

Timelapse fluorescence imaging of a GFP-tagged chimera (Fig. 2A and B) indicated that in contrast to control MF1 oocytes which normally degrade cyclin B between 8-11 h post-GVBD (Fig. 3A and C) (Herbert et al. 2003, Homer et al. 2005a), spindle depolymerisation inhibited degradation of cyclin B for $14 \mathrm{~h}$ (11 of 11 oocytes) (Fig. $3 \mathrm{~A}$ and $\mathrm{F}$ ) and beyond (data not shown). A previous report showed by Western blotting that a $3 \mathrm{~h}$ exposure to nocodazole stabilises cyclin B in mouse oocytes (Lefebvre et al. 2002). These data extend this observation by showing that stabilisation of cyclin B can be maintained for several hours upon spindle disruption. In keeping with inhibition of cyclin B destruction in nocodazole-treated oocytes (Fig. 3A), Cdk1 activity was stabilised during the period that $\mathrm{Cdk} 1$ inactivation was observed in controls (Fig. 3M and $\mathrm{N}$ ). This is consistent with data demonstrating that Cdk1 activity in meiosis I mouse oocytes is largely determined by levels of cyclin B (Hampl \& Eppig 1995, Polanski et al. 1998).

During mitosis, both securin and cyclin B are pivotal downstream targets of the SAC when the spindle is depolymerised (Michel et al. 2004). We were therefore interested in examining the fate of securin following spindle 
A

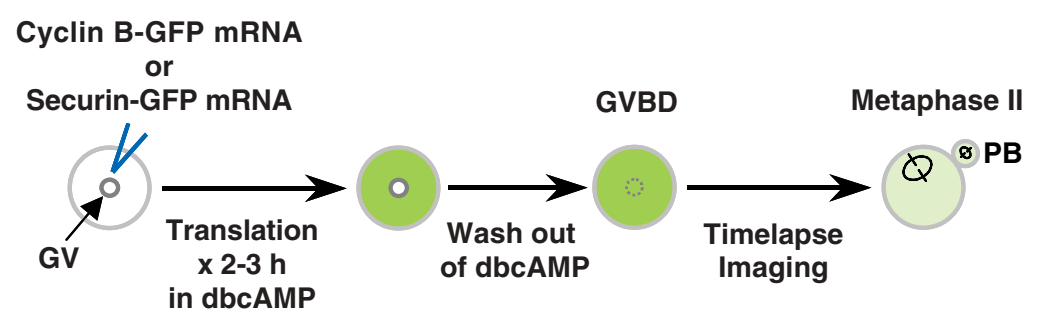

B

Cyclin B-GFP mRNA
or

Securin-GFP mRNA

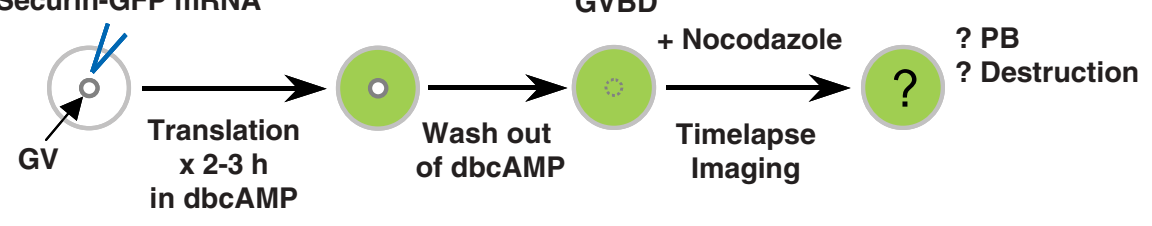

Figure 2 Schematics of experimental strategies. GV-stage MF1 oocytes were microinjected with $1 \mu \mathrm{g} / \mu \mathrm{l}$ of mRNAs encoding either securin-GFP or cyclin B-GFP and cultured in dibutyryl cyclic AMP

(dbcAMP)-treated medium for $\sim 2-3 \mathrm{~h}$ which prevents GVBD and allows time for translation. Oocytes were then washed out of dbcAMP to enable GVBD and cultured in either standard medium $(\mathrm{A})$ or nocodazole-treated medium (B) for the duration of timelapse fluorescence imaging whilst being maintained at $37^{\circ} \mathrm{C}$ in an atmosphere of $5 \% \mathrm{CO}_{2}$ on the microscope stage. depolymerisation. We found that unlike control oocytes which degrade securin with similar kinetics to cyclin B (Fig. $3 \mathrm{H}$ and J), nocodazole-treated oocytes stabilised securin for up to $14 \mathrm{~h}$ post-GVBD (7 of 7 oocytes) (Fig. $3 \mathrm{H}$ and L) and beyond (data not shown). Thus, spindle depolymerisation is associated with stabilisation of securin which persists for several hours.

We controlled for a number of factors which could have affected the interpretation of our results. Firstly, we found that the signal from a co-injected fluorescent reporter which is not metabolised, Texas Red (TR), remained stable (Fig. 3D and G), indicating that differences in GFP profiles represented true differences in turnover of GFP-tagged chimerae and were not due to artefacts in the imaging technique. Secondly, high levels of securin or cyclin B could potentially saturate the destruction machinery thereby inhibiting protein degradation and meiosis I (Ledan et al. 2001, Herbert et al. 2003, Terret et al. 2003, Marangos \& Carroll 2004). This was not the reason for meiosis I arrest in nocodazoletreated oocytes however as, compared with controls, drug-exposed oocytes translated either similar (Fig. 3A) or lower (Fig. $3 \mathrm{H}$ ) levels of protein by the time the destruction machinery became active in wild-type oocytes (8-9 h post-GVBD). Thirdly, it is theoretically possible that subtle amounts of protein destruction in nocodazole-treated oocytes might have been masked on a background of continuous protein synthesis. To address this, we clamped protein synthesis from injected mRNAs using a GFP-targeting antisense (Fig. 4A) as described previously (Nixon et al. 2002, Homer et al. 2005a). This showed that protein destruction was inhibited in nocodazole-treated oocytes even when protein levels were only $\sim 20-50 \%$ of the peak levels attained with mRNA alone (Fig. 4B and D). Inhibition of protein destruction at these lower protein levels further corroborates the assertion that saturation of the destruction machinery did not account for our observations. Finally, these are unlikely to be due to toxic nocodazole-specific effects since, in our culture conditions, MF1 oocytes retain the ability to reassemble a bipolar spindle and proceed to PBE by $\sim 3 \mathrm{~h}$ after removal from nocodazole $(92 \% ; n=50)$.

\section{Homologue disjunction does not occur during a prolonged meiosis I arrest}

During mitosis, prolongation of prometaphase by nocodazole provides additional time beyond that normally available during an unperturbed mitosis for the non-cleavage pathway to resolve arm cohesion completely (Uhlmann 2003, Giménez-Abián et al. 2004). Given that resolution of arm cohesion is responsible for homologue disjunction during meiosis I, one prediction is that if a non-cleavage mechanism operates in mouse oocytes then homologues would disjoin if prometaphase I is sufficiently prolonged. A previous report indicated that homologues did not disjoin in prometaphase I mouse oocytes exposed to $4 \mathrm{~h}$ of spindle depolymerisation (Brunet et al. 2003). However, since prometaphase I in mouse oocytes usually lasts $6-10 \mathrm{~h}$ (depending on the strain), this duration of nocodazole exposure may not have been long enough for the effects of a non-cleavage pathway to become cytologically evident.

In order to investigate this further, we examined oocytes that had been arrested in meiosis I for $>18 \mathrm{~h}$ post-GVBD. C-banded chromosome spreads of such oocytes showed that bivalents (recombined homologous pairs) persisted in all cases $(n=25)$ (Fig. 5A). The persistence of intact bivalents indicates that the recently reported loss of cohesin in prometaphase oocytes (Kouznetsova et al. 2005) is not sufficient for resolution of arm cohesion even during prolonged arrest. 
A

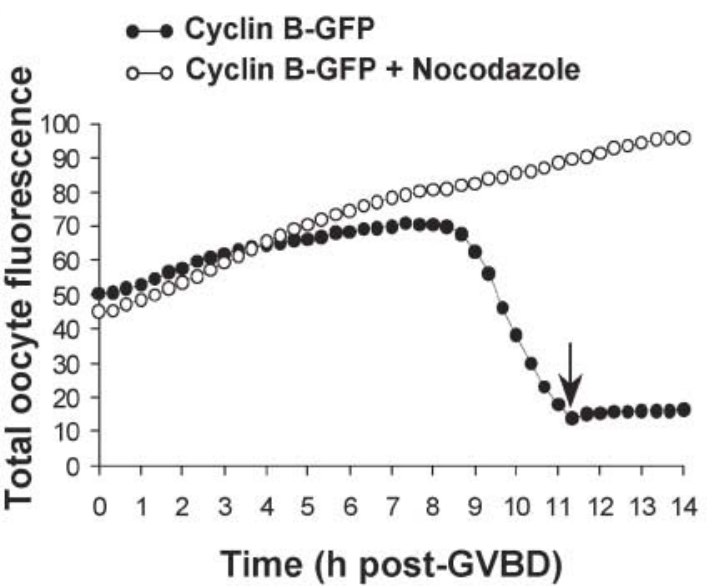

H

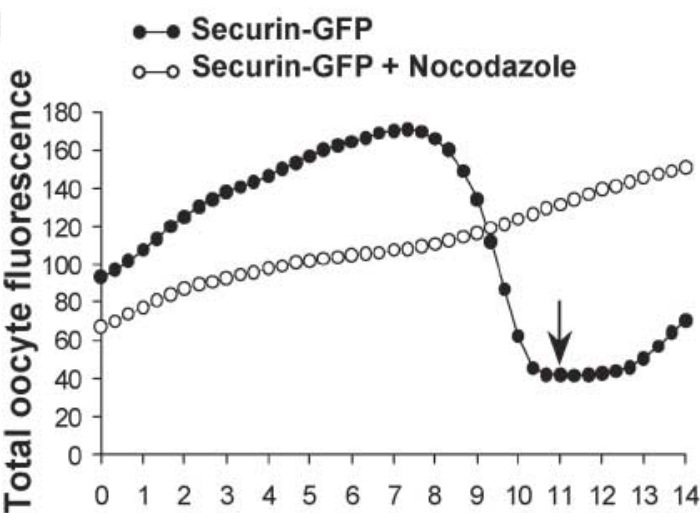

Time (h post-GVBD)

M

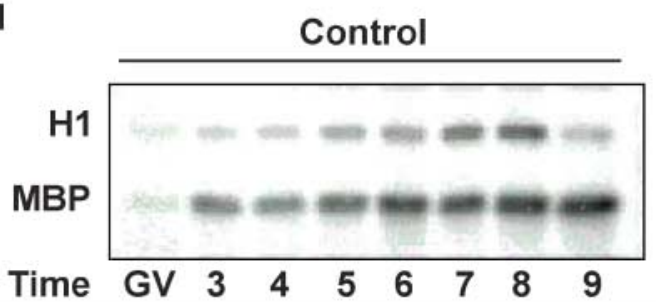

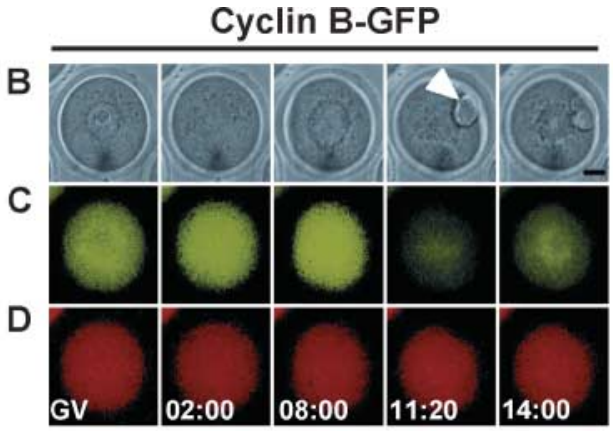
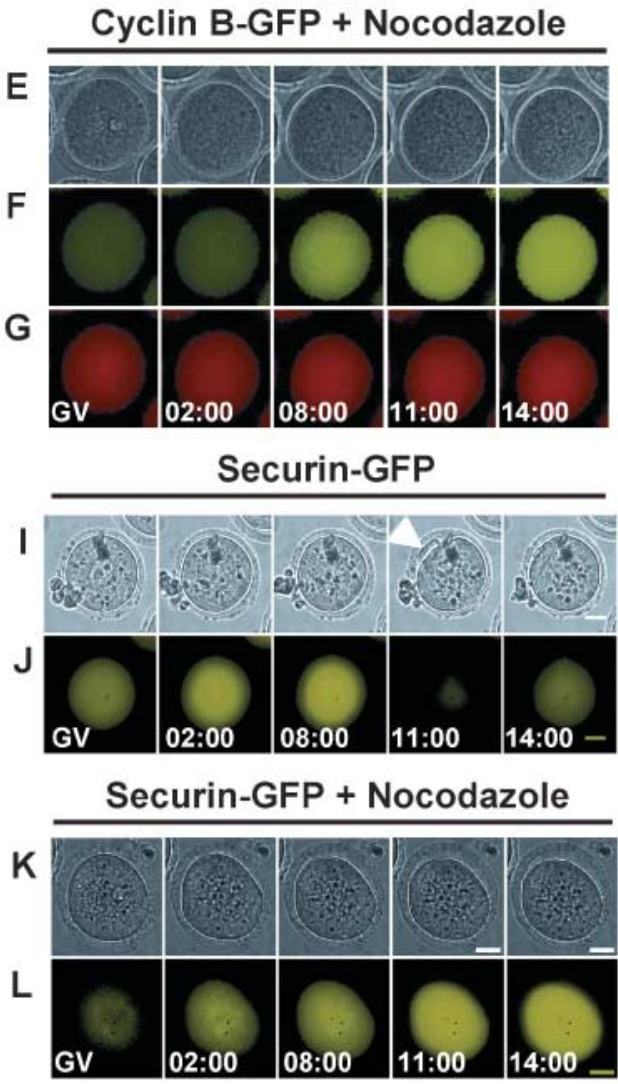

N

+ Nocodazole

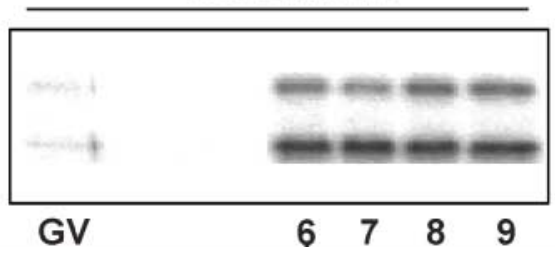

Figure 3 Spindle depolymerisation inhibits cyclin B and securin destruction, PBE and Cdk1 inactivation. (A to L) MF1 oocytes microinjected at the GV-stage with either cyclin B1-GFP mRNA and Texas Red (TR) (A to G) or securin-GFP mRNA (H to L) were examined by timelapse microscopy during culture in standard medium (A to D) and (H to J); or nocodazole-treated medium (A), (E), (F), (G), (H), (K) and (L). The profiles of representative individual oocytes are shown ( $\mathrm{A}$ and $\mathrm{H}$ ) with the time of PBE indicated by black arrows. Panels consist of brightfield (B), (E), (I) and (K); GFP fluorescence (C), (F), (J) and (L); and TR fluorescence (D) and (G) frames extracted from the individual timelapse series illustrated in $(\mathrm{A})$ and $(\mathrm{H})$ at the indicated times post-GVBD (in h:min). White arrowheads indicate PBs (B and I). Note the absence of PBs in nocodazole (E and K). Scale bars $=20 \mu \mathrm{m}$. ( $\mathrm{M}$ and $\mathrm{N}$ ) Histone $\mathrm{H} 1(\mathrm{H} 1)$ kinase and myelin basic protein (MBP) kinase activities in control (M) and nocodazoletreated (N) MF1 oocytes. Kinase assays reflect Cdk1 and MAPK activities as histone H1 and MBP are substrates of Cdk1 and MAPK respectively. MBP kinase acted as a loading control as, following activation at GVBD, MAPK activity remains stable during the meiosis I-to-meiosis II transition (see Polanski et al. 1998). Shown are representative segments of polyacylamide gels on which samples were run (3-8 experiments and 6 oocytes per lane). Time is in h post-GVBD. 
A

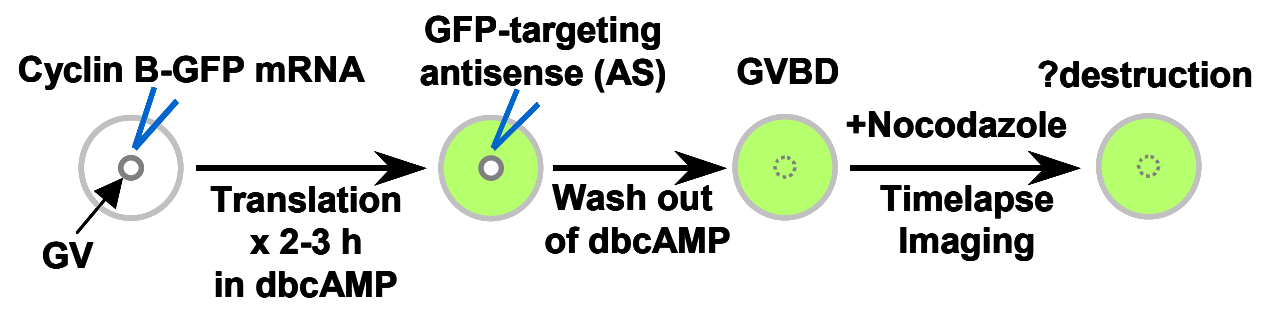

B
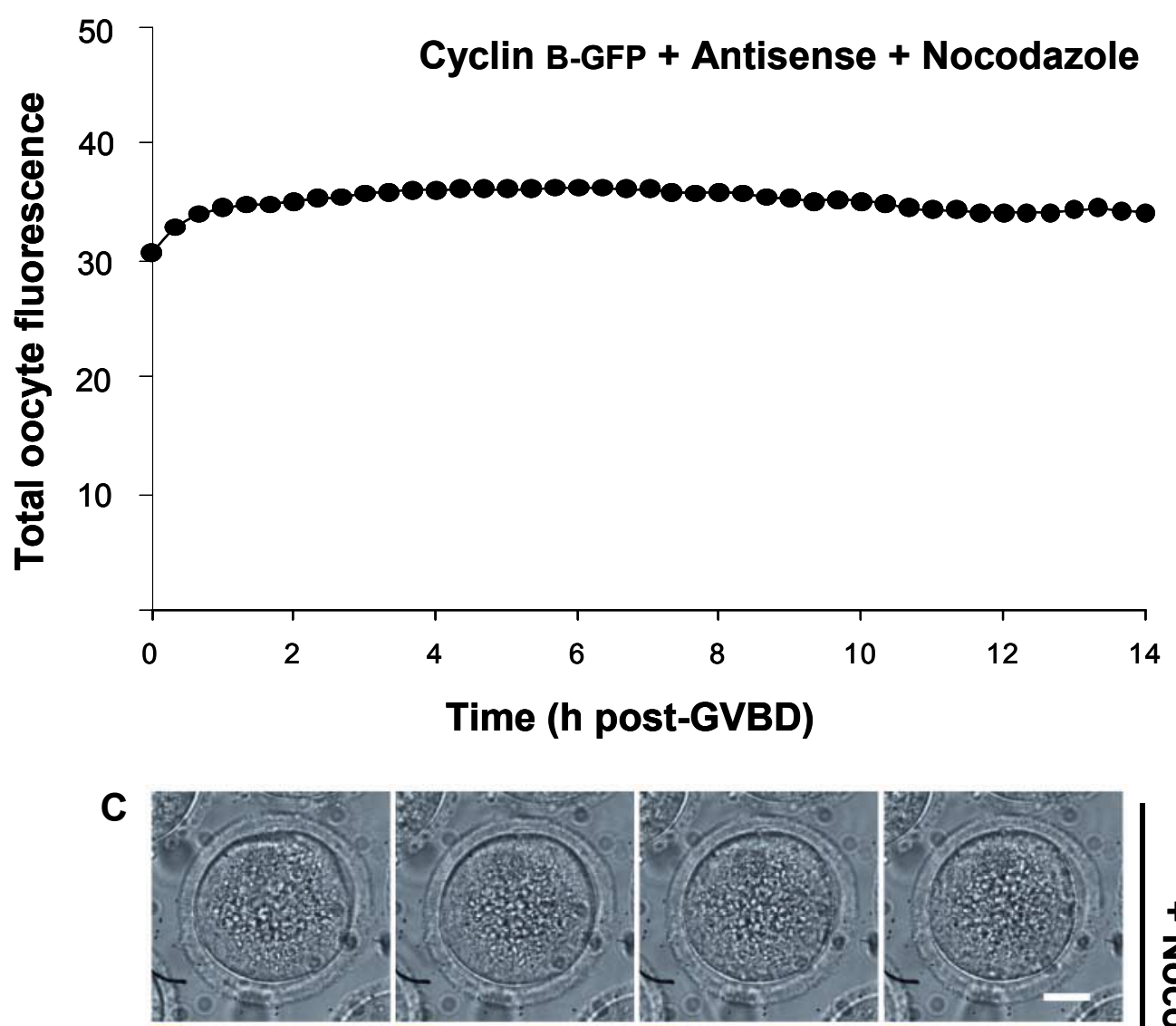

D
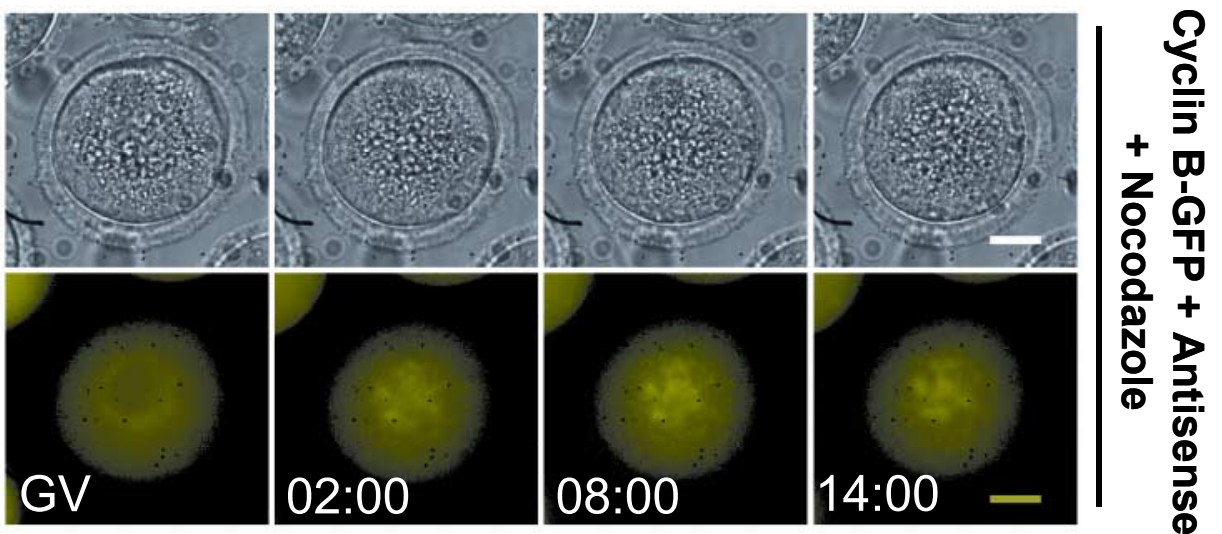

Figure 4 Cyclin B destruction is inhibited in Mad2-depleted oocytes expressing low and stable levels of cyclin B-GFP. (A) Experimental scheme for clamping protein synthesis. GV-stage oocytes were injected with cyclin B-GFP mRNA and cultured for 2-3 h in dbcAMP-treated medium to allow time for cyclin B-GFP to be translated. Oocytes were then injected with antisense designed to target GFP (AS) to arrest further cyclin B-GFP translation, washed out of dbcAMP to enable GVBD, and analysed by timelapse fluorescence microscopy during progression through meiosis I. (B to D) GV-stage oocytes injected with cyclin B-GFP mRNA $(n=7)$ followed by AS as depicted in (A) were examined by timelapse microscopy during culture in nocodazole-treated medium. The profile of a representative oocyte is shown (B) along with the corresponding brightfield (C) and fluorescence (D) frames at the indicated times post-GVBD in h:min. Note the absence of PBE by $14 \mathrm{~h}$ post-GVBD (C). Scale bars $=20 \mu \mathrm{m}$. 


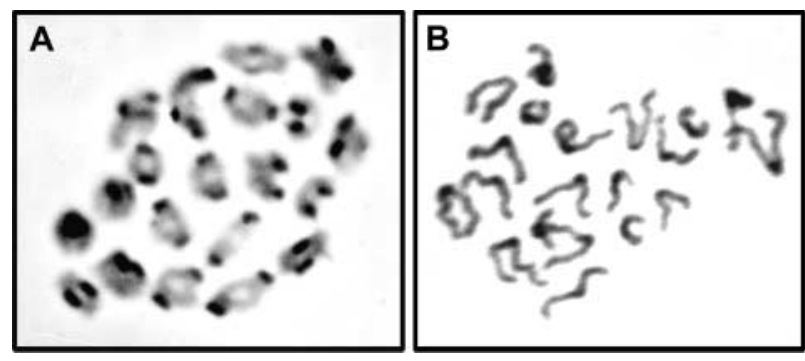

Figure 5 Homologues do not disjoin in nocodazole-treated oocytes. C-banded, air-dried chromosome spreads. Oocytes were cultured either in nocodazole-treated medium (A) or standard medium (B) for $18-20 \mathrm{~h}$ post-GVBD and then fixed and spread using alcohol:acetic acid. Chromatin was stained with Giemsa and centromeres were labelled with barium hydroxide. Shown is a representative spread of a nocodazole-treated mouse oocyte displaying 20 bivalents (A) and a representative spread of a control metaphase II-arrested oocyte displaying 20 univalents (B). Note that following loss of arm cohesion at anaphase I, the resulting univalents contain sister chromatids united only at centromeres. Because mouse chromosomes are acrocentric, this results in near complete separation of sister chromatids (B).

\section{Mad2 is required for the inhibition of cyclin $B$ and securin destruction and for inhibition of PBE following spindle depolymerisation}

We were interested in determining whether the meiosis I arrest produced by spindle depolymerisation was mediated by the SAC. In order to test this, we depleted Mad2 in MF1 oocytes utilising a Mad2-targeting morpholino oligonucleotide $(\mathrm{MO})$ designated $\mathrm{Mad} 2 \mathrm{MO}$ described previously (Fig. 6A) (Homer et al. 2005a). Western blotting indicated a substantial diminution of Mad2 levels that was specific to Mad2MO as a control $\mathrm{MO}$ which differed from Mad2MO by 5 bases ( 5 MispMO) did not affect Mad2 levels (Fig. 6B) (Homer et al. 2005a).

As before, we performed timelapse imaging of cyclin BGFP and securin-GFP and utilised the GFP-targeting antisense to increase our ability to detect any potential protein destruction (Fig. 7A). We found that none of the mockdepleted oocytes exhibited protein destruction $(n=12$; Fig. $7 \mathrm{~B}$ ) consistent with previous findings in the absence of MOs (see Fig. 4). However, following Mad2 depletion, cyclin B-GFP and securin-GFP underwent partial destruction in 7 of $10(70 \%)$ and 6 of $11(55 \%)$ oocytes respectively (Fig. 7B, D and F). The observed decline in GFP fluorescence was due to targeting of securin and cyclin B as Mad2-depleted oocytes injected with mRNA encoding GFP by itself did not exhibit a decline in fluorescence when compared with oocytes injected with cyclin B-GFP mRNA (Fig. 8A). Furthermore, unlike wild-type oocytes which never extruded a PB in nocodazole, 10 of $65(15 \%)$ of Mad2-depleted oocytes either underwent PBE (Fig. 8A and $\mathrm{B}$, oocytes 1 and 2) or produced multiple PB-like cytoplasmic blebs (Fig. 8B, oocyte 3). These PBs were devoid of DNA likely due to the absence of a spindle (Fig. 8). We were interested in determining whether homologues within this single group of chromatin had
A

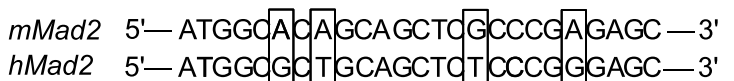

B

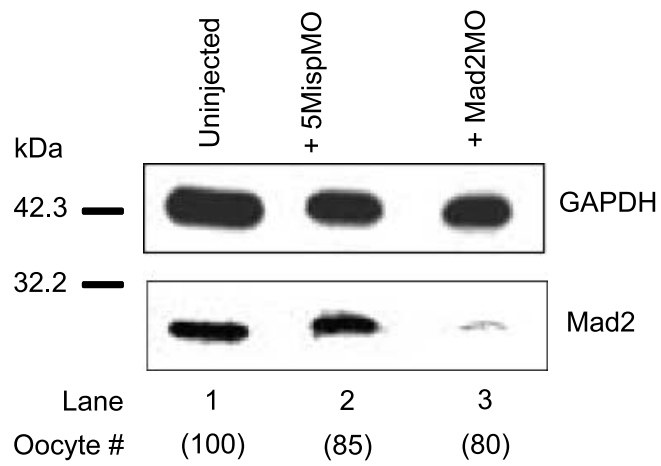

Figure $6 \mathrm{Mad} 2 \mathrm{MO}$ depletes the majority of Mad2 in mouse oocytes. (A) Nucleotide sequence of the start region of murine Mad2a (mMad2) (GenBank accession no. AF261919, Dobles et al. 2000) that Mad2MO was designed to target along with the aligned sequence of human Mad2 (hMad2) (GenBank accession no.U65410; $\mathrm{Li}$ and Benezra 1996). Differences in nucleotide sequences are highlighted by boxes. (B) Mouse oocytes microinjected with either a control MO (5MispMO; lane 2) or Mad2MO (lane 3) at the GV-stage were immunoblotted for Mad2 alongside uninjected oocytes about $16 \mathrm{~h}$ post-GVBD after they had completed meiosis I. GAPDH served as a loading control.

disjoined as might be expected due to the destabilisation of securin. We found that bivalents of Mad2-depleted oocytes remained intact even in oocytes that had extruded PBs in the presence of nocodazole (data not shown). This may have been due to incomplete destruction of securin or a requirement for spindle microtubules to physically pull homologues apart.

In order to provide a more objective and quantitative assessment of the degree of protein destruction in Mad2 depleted oocytes, we performed statistical comparisons between fluorescence intensities at the time of GVBD and at $20 \mathrm{~h}$ post-GVBD. Owing to the fact that we were working with relatively low fluorescence levels as a result of clamping of protein synthesis by GFP antisense, we were concerned that variations, albeit minor, in fluorescence capture could artefactually distort results. To eliminate this possibility we normalized GFP fluoresence intensity to the fluorescence intensity from a fixed concentration of TR added to the injection cocktail. We found that the normalized fluorescence (GFP:TR ratio) was not significantly altered in oocytes injected with cyclin B-GFP mRNA alone between the time of GVBD and $20 \mathrm{~h}$ post-GVBD (Fig. 9A) consistent with previous results (see Fig. 4). In contrast, the normalized fluorescence declined significantly in Mad2-depleted oocytes over the same time period (Fig. 9B). These differences were specifically due to Mad2 depletion as firstly, two control morpholinos had no effect on normalized fluorescence intensities (Fig. 9C and D). Secondly, complementation studies with a GFP-tagged 
A mRNA +

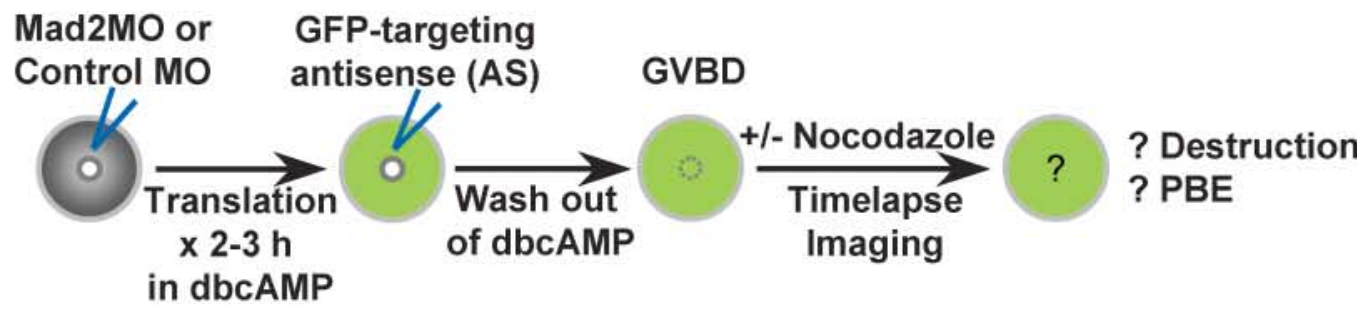

B

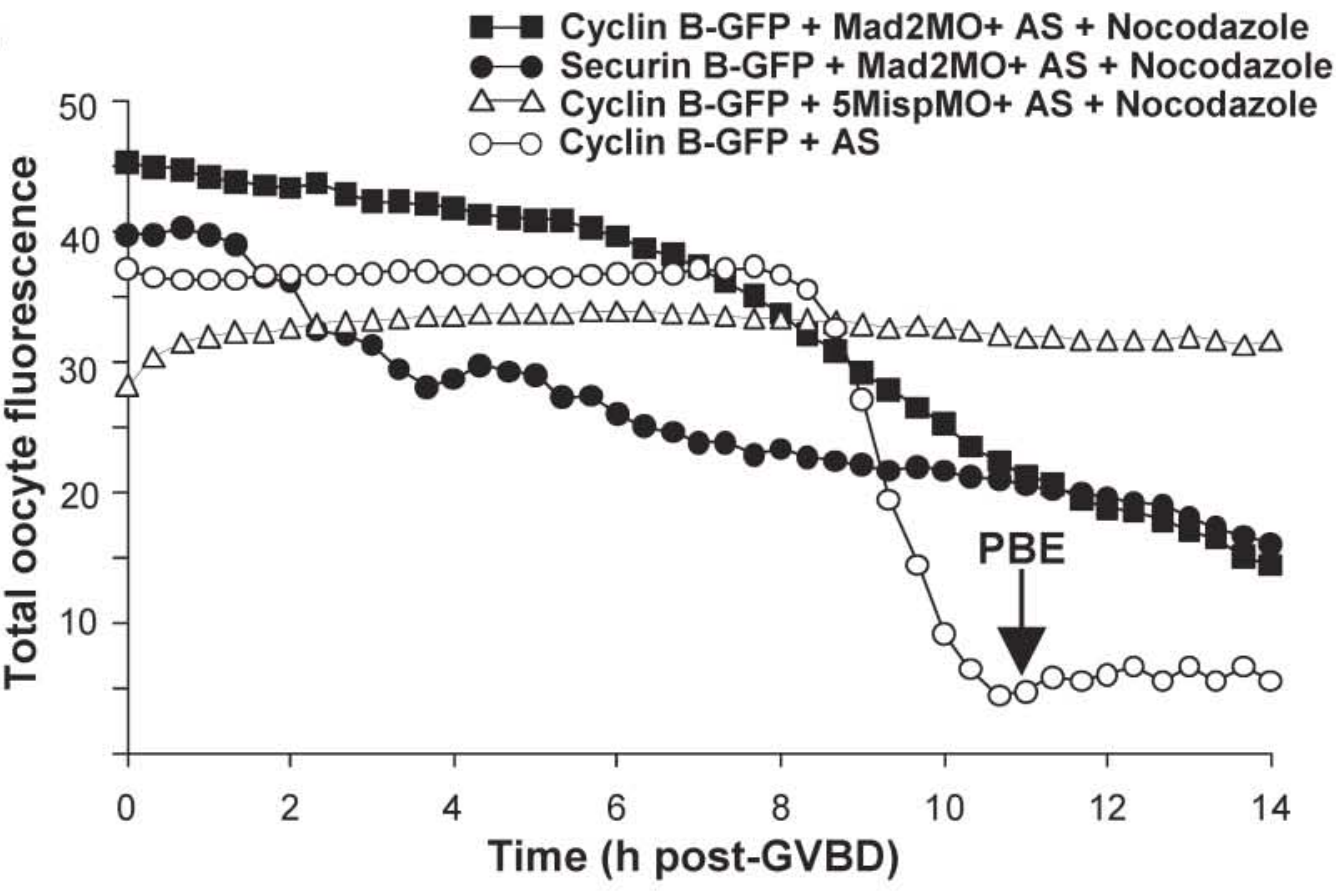

C
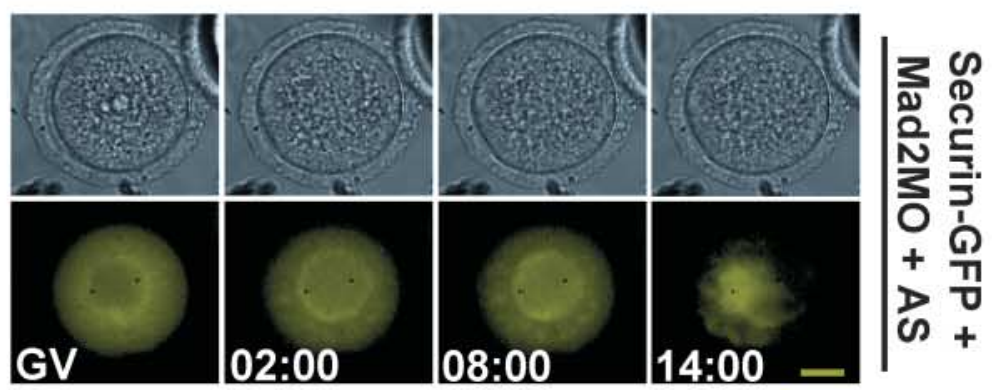

E
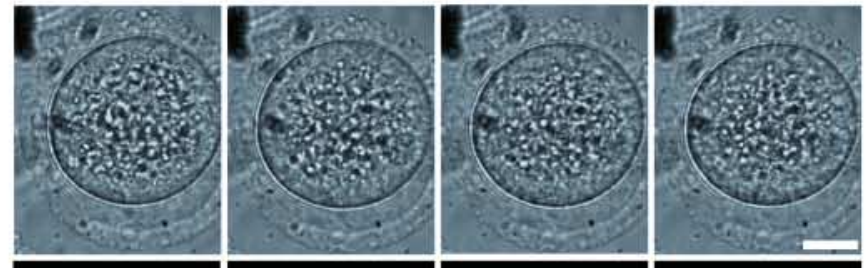

$\mathbf{F}$
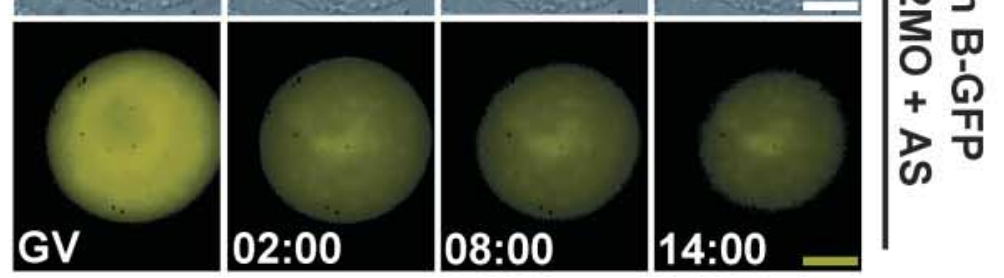
hMad2 construct which has previously been used in mouse oocytes by ourselves and others (Wassmann et al. 2003, Homer et al. 2005a, Homer et al. 2005b), revealed that hMad2 efficiently rescued mMad2-depleted oocytes (Fig. 9E) due to sequence differences in the start region between hMad2 and mMad2 (Fig. 6A), rendering hMad2 resistant to Mad2MO.

Finally we tried to gain more insight into the mechanism of protein degradation in Mad2 depleted oocytes. The instability of securin and cyclin B in Mad2 depleted oocytes exposed to nocodazole could be accounted for by unrestrained $\mathrm{APC} / \mathrm{C}$ activity. If this were so, then one would expect the APC/C degradation motif in cyclin B, the D-box, to be important for the observed protein degradation (Hagting et al. 2002, Castro et al. 2005). In order to test this we examined Mad2 depleted oocytes injected with $\Delta 90$ Cyclin B-GFP mRNA which lacks a D-box. We found that $\Delta 90$ Cyclin B-GFP remained stable in Mad2 depleted oocytes in the presence of nocodazole based on stable normalized fluorescence intensities (Fig. 9F) thereby supporting a requirement for the D-box and hence the APC/C in mediating protein destruction in Mad2 depleted oocytes.

\section{Discussion}

\section{Characterising the meiosis I arrest induced by spindle depolymerisation in mouse oocytes}

It was important to examine the effect of Mad2 on both securin and cyclin B to more fully characterise the nature of the molecular details underpinning the meiosis I arrest of mouse oocytes exposed to spindle depolymerisation. Firstly, the role of Mad2 following spindle depolymerisation was hitherto unknown. Secondly, securin destruction can be uncoupled from that of cyclin B (Michel et al. 2004). That this could also occur in mouse oocytes is suggested by the fact that homologues can and do disjoin whilst apparently arrested in meiosis I by nocodazole (Shen et al. 2005). In this regard, securin is a notable omission from prior studies involving mouse oocytes and spindle poisons. Thirdly, the one previous study to address the role of Mad2 in mouse oocytes exposed to spindle poisons provided only indirect evidence (in the form of Cdk1 activity) regarding the fate of cyclin B (Wassmann et al. 2003). In the absence of incontrovertible proof however, it is tenuous to regard Cdk1 activity as prima facie evidence of cyclin B turnover as data from two mammalian systems closely related to mouse oocytes, pig oocytes and rat embryos, indicate that Cdk 1 activity is not necessarily correlated with cyclin B levels (Josefsberg et al. 2001, Takakura et al. 2005).

Here we show that the meiosis I arrest of mouse oocytes following spindle depolymerisation is mediated by a Mad2-dependent response which inhibits destruction of both securin and cyclin B. Importantly, the robust SACmediated meiosis I arrest we observed suggests that the transient delay in the face of low doses of nocodazole (Wassmann et al. 2003) does not represent an inherently leaky SAC network in mammalian oocytes. Instead, as we discuss next, these responses are likely to reflect differences in kinetochore-microtubule defects induced by different doses of spindle poisons.

\section{The type of spindle defect incurred during meiosis I determines whether or not mouse oocytes will sustain a prolonged $S A C$ response}

We found that spindle depolymerisation by $5 \mu \mathrm{M}$ nocodazole during meiosis I in mouse oocytes elicited a robust SAC-mediated arrest lasting over $18 \mathrm{~h}$. At odds with our results, chronic exposure to nanomolar concentrations of nocodazole which leave an intact spindle lead to 40-60\% PBE rates after a transient delay (Wassmann et al. 2003, Shen et al. 2005). Our results are not the result of toxicity due to higher drug dosage as timelapse imaging confirmed long-term viability along with a capacity for continued protein synthesis amongst oocytes exposed to prolonged pharmacological treatment (see Fig. 3). Furthermore, wild-type levels of PBE were observed following transfer to drug-free medium. It seems unlikely that the initial defect in low doses of nocodazole was rectified during the transient delay as such oocytes experience elevated rates of chromosome missegregation (Shen et al. 2005) and, in keeping with this, about half go on to exhibit misaligned chromosomes at the metaphase II stage (Wassmann et al. 2003). Rather, it appears that although mouse oocytes mount an SAC response to varying types of spindle disruption, the SAC is susceptible to 'slippage' when faced with certain types of defects. We propose two possible explanations (that are not mutually exclusive) which seek to differentiate between quantitative and qualitative aspects of the defects.

From a 'quantitative' perspective, it may be that defects in kinetochore attachment/tension produced by low doses of nocodazole were simply too subtle to sustain a prolonged meiosis I arrest. In contrast, the complete absence of tension and attachment resulting from spindle

Figure $7 \mathrm{Mad} 2$ is required for inhibiting securin and cyclin B destruction following spindle depolymerisation. (A) Scheme employing AS-based clamping of translation from cyclin B-GFP and securin-GFP mRNAs as described in Fig. 4A incorporating morpholinos. (B to F) GV-stage oocytes coinjected with securin-GFP mRNA + Mad2MO; cyclin B-GFP mRNA + Mad2MO; or cyclin B-GFP mRNA + 5MispMO (mock-depleted) followed by AS as in (A) were examined by timelapse microscopy during culture in nocodazole. To enable a comparison of protein degradation profiles in the presence and absence of a spindle, an oocyte injected with cyclin B-GFP mRNA + AS which was analysed by timelapse microscopy during culture in standard medium (spindle intact) is also shown. Shown are profiles of representative oocytes (B) and corresponding brightfield ( $\mathrm{C}$ and $\mathrm{E}$ ) and fluorescence frames ( $\mathrm{D}$ and $\mathrm{F}$ ) for Mad2 depleted oocytes at the indicated times post-GVBD (h:min). Scale bars $=20 \mu \mathrm{m}$. 


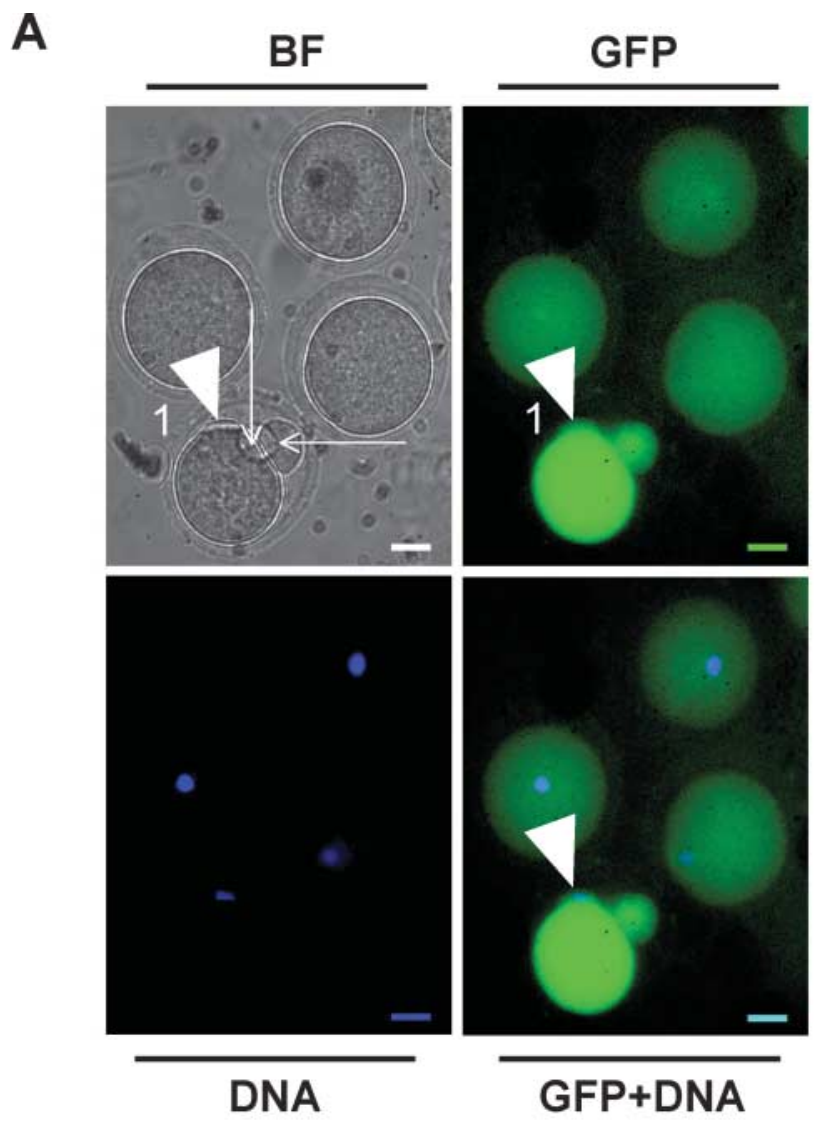

B
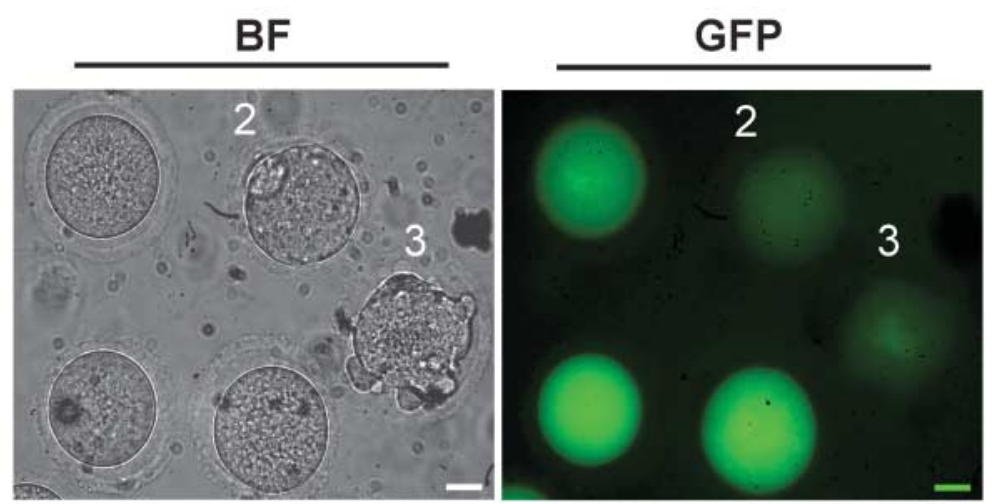

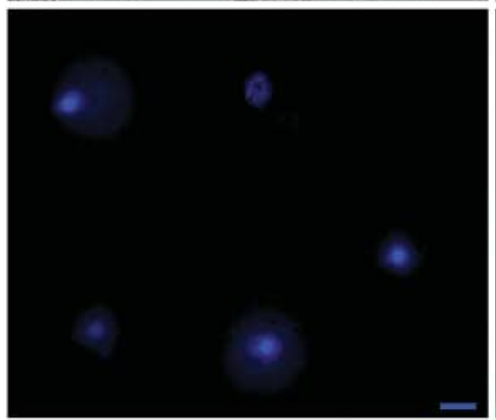

DNA

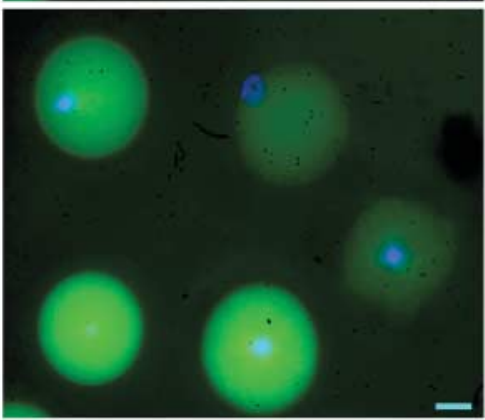

GFP+DNA
Figure $8 \mathrm{Mad} 2$ depleted oocytes undergo PBE in nocodazole and cyclin B-GFP but not GFP is degraded following Mad2 knockdown. (A) Mad2 knockdown results in PBE in nocodazole and GFP alone is not destroyed in Mad2 depleted oocytes. GV oocytes were coinjected with Mad2MO and either GFP mRNA (oocyte $1 ; n=7$ ) or cyclin BGFP mRNA (un-numbered oocytes; $n=6$ ) followed by AS as described in Fig. 7A and cultured in nocodazole. Representative oocytes which possessed similar GFP fluorescence at the commencement of culture are shown after culture for 18-20 h postGVBD. Hoechst 33342-stained DNA appears in blue. Arrows point to each of two PBs and arrowhead points to a peripheral 'pocket' in $2^{\circ}$ oocyte containing DNA. (B) Mad2 knockdown results in PBE in nocodazole. GV oocytes were injected either with cyclin B1-GFPmRNA + AS (un-numbered oocytes; $n=5$ ) or with cyclin B1-GFPmRNA + Mad2MO + AS (oocytes $2 \& 3 ; n=10$ ) and cultured in nocodazole. Representative oocytes which expressed similar levels of cyclin B1-GFP at the commencement of culture are shown after culture for $18-20 \mathrm{~h}$ post-GVBD. Hoechst 33342-stained DNA appears in blue. Scale bars $=20 \mu \mathrm{M}$. 


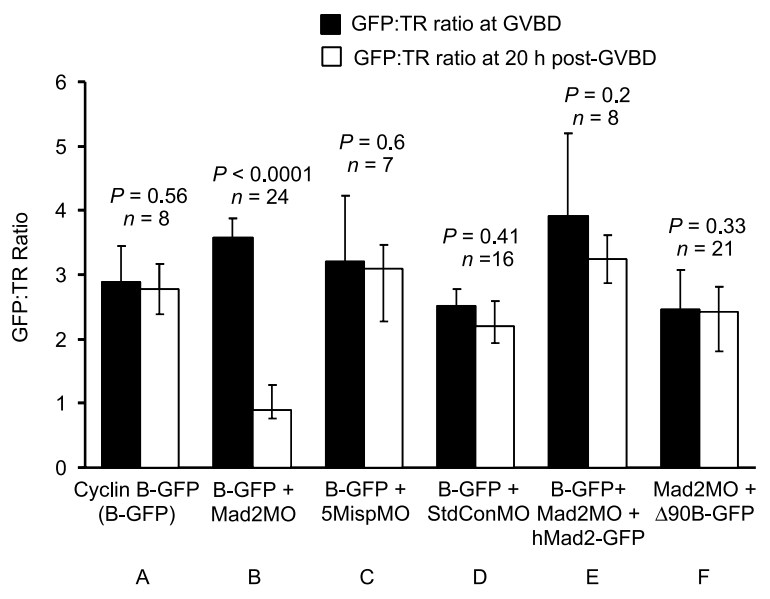

Figure 9 Semi-quantitative analyses of the degree of protein destruction. (A to D). Mad2 depleted oocytes but not mock-depleted oocytes degrade cyclin B in nocodazole. GV-stage oocytes were injected with cyclin B-GFP mRNA + TR (A) or with cyclin B-GFP mRNA along with one of Mad2MO (B), 5MispMO (C) or StdConMO (D) and TR followed by antisense as described and followed by timelapse microscopy during culture in nocodazole-treated medium. (E) Destruction of cyclin B-GFP in oocytes depleted of endogenous mMad2 can be rescued by hMad2-GFP. GV-stage oocytes were injected with cyclin B-GFP mRNA + Mad2MO + hMad2-GFP mRNA + TR + AS as described and followed by timelapse microscopy. (F) Cyclin B-GFP destruction in Mad2 depleted oocytes is dependent upon the $\mathrm{D}$ box (see main text for further details). GVstage oocytes were injected with $\Delta 90$ cyclin B-GFP mRNA + Mad2MO + TR + AS as described and followed by timelapse microscopy. In all experimental groups, GFP and TR fluorescence intensities were quantified for each oocyte around the time of GVBD and at $20 \mathrm{~h}$ post-GVBD from which the GFP:TR ratios were determined. Data is shown as the mean \pm S.E.M.s. Oocyte numbers and $P$ values derived using the Student's $t$-test are depicted above each experimental group.

depolymerisation generated a cumulatively stronger signal capable of producing a robust SAC-mediated arrest. Whilst these differences may be inconsequential to the durability of the SAC response within the relatively small volume of somatic cells (Skoufias et al. 2001), signal strength is likely to be significant in the $\sim 40$-fold larger volume of the mouse oocyte. This might account for the failure of one or a few misaligned chromosomes to induce a meiosis I delay/arrest (LeMaire-Adkins et al. 1997) whereas oocytes of the $\mathrm{Mlh} 1^{-1-}$ mouse consistently arrest at prometaphase I when, due to a recombination defect, the majority of chromosomes fail to align (Woods et al. 1999).

Alternatively, if tension is the primary defect in mouse oocytes exposed to low doses of spindle poisons (Wassmann et al. 2003) as in mitotic cells under similar experimental conditions (Skoufias et al. 2001), then current observations could reflect a poorly developed tension-sensing arm of the SAC in mammalian oocytes. During meiosis I, tension is usually generated when the pulling force of microtubules exerted on a bivalent with bipolar orientation is resisted by arm cohesion distal to chiasmata
(Watanabe 2004). However, this latter 'qualitative' argument seems contrary to prior reports demonstrating that tension is especially crucial for satisfying the SAC in many meiosis I systems including yeast, mantid spermatocytes and maize (Li \& Nicklas 1995, Yu et al. 1999, Shonn et al. 2000). Furthermore, lack of a tension-sensing SAC in mouse oocytes would be inconsistent with the extended prometaphase I arrest (18-20 h) sustained by over $80 \%$ of Mlh1 $1^{-1-}$ oocytes (Woods et al. 1999). In these oocytes, the majority of homologous chromosomes fail to recombine and thus attain monopolar attachments resulting in a spindle apparatus lacking in tension (Woods et al. 1999).

\section{Implications for the roles of the APC/C and the meiotic spindle during female mammalian meiosis I}

During mitosis, the ability of Mad2 to regulate the destruction of securin and cyclin $\mathrm{B}$ is reliant on its ability to modulate APC/C activity and the ability of the APC/C to orchestrate the destruction of its substrates is dependent on them possessing conserved recognitions motifs such as the D-box (Musacchio \& Hardwick 2002, Taylor et al. 2004, Castro et al. 2005). As a consequence of this, when Mad2 is depleted during mitosis, securin and cyclin B destruction occur prematurely thereby curtailing mitosis, securin and cyclin B are destabilised in the absence of a spindle (Michel et al. 2004). Conversely, Mad2 overexpression induces a metaphase arrest (Howell et al. 2000), presumably due to APC/C inhibition.

Collectively, the data presented here along with previous reports in mouse oocytes conform to the mitotic model and therefore strongly support a pivotal role for the APC/C during female mammalian meiosis I. Firstly, Mad2 is required for stabilising securin and cyclin B following spindle depolymerisation (this paper) and degradation of securin and cyclin B occur prematurely in untreated oocytes in which Mad2 is depleted (Homer et al. 2005a). Consistent with this, Mad2 $\Delta \mathrm{C}$ which is unable to sequester Cdc20 and two SAC-resistant Cdc20 mutants with dominant negative activity shortened the duration of meiosis I in mouse oocytes (Tsurumi et al. 2004). Secondly, D-box mutations/deletions in securin and cyclin B stabilise both proteins in untreated mouse oocytes and inhibit homologue disjunction and PBE (Herbert et al. 2003). Thirdly, although full-length cyclin B-GFP was unstable in Mad2 depleted oocytes treated with nocodazole, $\Delta 90$ cyclin B-GFP remained stable (this paper). In other words, elimination of the APC/C substrate-targeting motif now renders that substrate $(\Delta 90$ cyclin B) resistant to degradation even when the APC/C is expected to be active. Finally, measured overexpression of Mad2 induces a dose-dependent metaphase I arrest in mouse oocytes (Wassmann et al. 2003, Homer et al. 2005, Homer et al. 2005b).

Notably, securin and cyclin B were degraded to a lesser extent in Mad2-depleted oocytes following spindle disruption when compared with untreated oocytes in which the 
spindle was present (see Fig. 7B). Reduced securin and cyclin B degradation in Mad2 depleted oocytes might be the consequence of small residual levels of Mad2 or might reflect the activity of other SAC proteins such as Bub1 or BubR1 (Brunet et al. 2003, Tsurumi et al. 2004). Another possible explanation is that the spindle is required for efficient APC/C-mediated substrate degradation. In support of this notion, the proteasome localises to the spindle apparatus in rat oocytes (Josefsberg et al. 2000) and Cdc20, cyclin B and securin have all been shown to localise to the spindle in mouse oocytes (Marangos \& Carroll 2004, Tsurumi et al. 2004, our unpublished data). Furthermore, artificial induction of intracellular calcium transients by ionophores, efficiently triggered homologue disjunction in metaphase l-arrested oocytes but only if an intact spindle was present (Soewarto et al. 1995). This might explain why disabling Mad2 in nocodazole-treated oocytes possessing an intact spindle led to PBs containing DNA (Wassmann et al. 2003) whereas in the absence of a spindle, PBs were devoid of DNA and homologues did not disjoin (this paper). Indeed, it has been proposed that chromosomes may be physically separated by the shearing forces of the spindle in mouse oocytes in spite of separase inhibition when cohesin would be expected to remain intact (Terret et al. 2003).

\section{Homologues do not disjoin in nocodazole: insights into the regulation of arm cohesion during mammalian meiosis I}

During meiosis I, homologue disjunction is brought about through the resolution of arm cohesion distal to cross-over sites (Watanabe 2004). During vertebrate mitosis, a noncleavage pathway removes the bulk of arm cohesins during prometaphase (Uhlmann 2003). If a similar pathway operates during meiosis I in mammalian oocytes then one might expect homologues to disjoin completely during a prolonged nocodazole-induced arrest as this would provide time for any cleavage-independent pathway to completely remove arm cohesins.

To investigate this possibility, we cultured mouse oocytes in nocodazole-treated medium and performed airdried spreads about $18-20 \mathrm{~h}$ post-GVBD which is about twice as long as the duration of meiosis I in wild-type MF1 oocytes. Centromere labelling using the technique of C-banding enabled us to unambiguously differentiate bivalent, univalent and single chromatid morphologies amongst chromosome spreads (Homer et al. 2005a). Together, this combined approach enabled an indirect assessment of the effect of prolonged prometaphase I arrest on arm cohesion. We found that bivalents remained intact after a prolonged prometaphase I arrest induced by nocodazole indicating that arm cohesion remained intact. From this we can conclude that a cleavage-independent pathway is not sufficient on its own for resolving all arm cohesion during mammalian meiosis I. This is in keeping with the requirement for securin destruction (Herbert et al.
2003) and separase activity (Terret et al. 2003) for the metaphase I to anaphase I transition in mouse oocytes.

The data presented here indicate that mammalian oocytes employ much of the same machinery as mitotic cells for executing a robust meiosis I arrest upon spindle depolymerisation. Specifically, Mad2 is indispensable and two of the primary downstream targets are securin and cyclin B. In the future it will be important to explore the contributions made by defects in tension and attachment towards the activation and maintenance of the SAC in mammalian oocytes as well as the involvement of other SAC proteins in sensing and/or correcting such defects.

\section{Acknowledgements}

We thank J Pines, J Pinto-Toro and R Benezra for the generous gifts of plasmids. We acknowledge $\mathrm{K}$ Lacsko and all members of the laboratory for technical assistance and advice. This work was supported by a WellBeing Research Training Fellowship (RTF/387) to HA Homer and grants from Newcastle University Hospitals Special Trustees and Wellcome to M Herbert and A McDougal.

\section{References}

Brunet S, Santa Maria A, Guillaud P, Dujardin D, Kubiak JZ \& Maro B 1999 Kinetochore fibers are not involved in the formation of the first meiotic spindle of mouse oocytes, but control the exit from the first meiotic M phase. Journal of Cell Biology 146 1-12.

Brunet S, Pahlavan G, Taylor SS \& Maro B 2003 Functionality of the spindle checkpoint during the first meiotic division of mammalian oocytes. Reproduction $126443-450$.

Castro A, Bernis C, Vigneron S, Labbé J \& Lorca T 2005 The anaphase-promoting complex: a key factor in the regulation of cell cycle. Oncogene 24 314-325.

Dobles M, Liberal V, Scott ML, Benezra R \& Sorger PK 2000 Chromosome missegregation and apoptosis in mice lacking the mitotic checkpoint protein Mad2. Cell 101 635-645.

Giménez-Abián J, Sumara I, Hirota T, Hauf S, Gerlich D, de la Torre C, Ellenberg J \& Peters J 2004 Regulation of sister chromatid cohesion between chromosome arms. Current Biology 14 1187-1193.

Hagting A, den Elzen N, Vodermaier HC, Waizenegger IC, Peters J-M \& Pines J 2002 Human securin proteolysis is controlled by the spindle checkpoint and reveals when the APC/C switches from activation by Cdc20 to Cdh1. Journal of Cell Biology 157 $1125-1127$.

Hampl A \& Eppig JJ 1995 Analysis of the mechanism(s) of metaphase I arrest in maturing mouse oocytes. Development 121 925-933.

Herbert M, Levasseur $M$, Homer $H$, Yallop $K$, Murdoch A \& McDougall A 2003 Homologue disjunction in mouse oocytes requires proteolysis of securin and cyclin B1. Nature Cell Biology 5 1023-1025.

Homer H, McDougall A, Levasseur M, Yallop K, Murdoch A \& Herbert M 2005a Mad2 prevents aneuploidy and premature proteolysis of cyclin B and securin during meiosis I in mouse oocytes. Genes and Development 19 202-207.

Homer H, McDougall A, Levasseur M, Murdoch A \& Herbert M 2005b RNA iterference in human oocytes: towards an understanding of human aneuploidy. Molecular Human Reproduction $11397-404$.

Howell BJ, Hoffman DB, Fang G, Murray AW \& Salmon ED 2000 Visualization of Mad2 dynamics at kinetochores, along spindle fibers, and at spindle poles in living cells. Journal of Cell Biology $1501233-1249$ 
Josefsberg L, Galiani D, Dantes A, Amsterdam A \& Dekel N 2000 The proteasome is involved in the first metaphase-to-anaphase transition of meiosis in rat oocytes. Biology of Reproduction 62 1270-1277.

Josefsberg L, Kaufman O, Galiani D, Kovo M \& Dekel N 2001 Inactivation of $\mathrm{M}$-phase promoting factor at exit from first embryonic mitosis in the rat is independent of cyclin B1 degradation. Biology of Reproduction $64871-878$.

Kouznetsova A, Novak I, Jessberger R \& Höög C 2005 SYCP2 and SYCP3 are required for cohesin core integrity at diplotene but not for centromere cohesion at the first meiotic division. Journal of Cell Science 118 2271-2278.

Ledan E, Polanski Z, Terret M-E \& Maro B 2001 Meiotic maturation of the mouse oocyte requires an equilibrium between cyclin $B$ synthesis and degradation. Developmental Biology 232 400-413.

Lefebvre C, Terret ME, Dijane A, Rassinier P, Maro B \& Verlhac M-H 2002 Meiotic spindle stability depends on MAPK-interacting and spindle-stabilizing (MISS), a new MAPK substrate. Journal of Cell Biology 157 603-613.

LeMaire-Adkins R, Radke K \& Hunt PA 1997 Lack of checkpoint control at the metaphase/anaphase transition: a mechanism of meiotic nondisjunction in mammalian females. Journal of Cell Biology $1391611-1619$.

Levasseur M \& McDougall A 2000 Sperm-induced calcium oscillations at fertilisation in ascidians are controlled by cyclin B1-dependent kinase activity. Development 127 631-641.

Li X \& Nicklas BR 1995 Mitotic forces control a cell cycle checkpoint. Nature 373 630-632.

Li Y \& Benezra R 1996 Identification of a human mitotic checkpoint gene: hsMAD2. Science 274 246-248.

Marangos P \& Carroll J 2004 The dynamics of cyclin B1 distribution during meiosis I in mouse oocytes. Reproduction 128 153-162.

McDougall A \& Levasseur M 1998 Sperm-triggered calcium oscillations during meiosis in ascidian oocytes first pause, restart, then stop: correlations with cell cycle kinase activity. Development 125 4451-4459.

Meraldi P, Draviam V \& Sorger P 2004 Timing and checkpoints in the regulation of mitotic progression. Developmental Cell 7 45-60.

Michel L, Diaz-Rodriguez E, Narayan G, Hernando E, Murty V \& Benezra R 2004 Complete loss of the tumor suppressor MAD2 causes premature cyclin B degradation and mitotic failure in human somatic cells. PNAS 101 4459-4464.

Morrow C, Tighe A, Johnson V, Scott M, Ditchfield C \& Taylor S 2005 Bub1 and aurora B cooperate to maintain BubR1-mediated inhibition of APC/CCdc20. Journal of Cell Science 118 3639-3652.

Musacchio A \& Hardwick KG 2002 The spindle checkpoint: structural insights into dynamic signalling. Nature Reviews Molecular Cell Biology 3 731-741.

Nixon VL, Levasseur M, McDougall A \& Jones KT $2002 \mathrm{Ca}^{2+}$ oscillations promote APC/C-dependent cyclin B1 degradation during metaphase arrest and completion of meiosis in fertilizing mouse eggs. Current Biology 12 746-750.

Polanski Z, Ledan E, Brunet S, Louvet S, Verlhac M-H, Kubiak JZ \& Maro B 1998 Cyclin synthesis controls the progression of meiotic maturation in mouse oocytes. Development 125 4989-4997.

Shen Y, Betzendahl I, Sun F, Tinneberg H \& Eichenlaub-Ritter U 2005 Non-invasive method to assess genotoxicity of nocodazole interfering with spindle formation in mammalian oocytes. Reproductive Toxicology 19 459-471.

Shonn M, McCarroll R \& Murray AW 2000 Requirement of the spindle checkpoint for proper chromosome segregation in budding yeast meiosis. Science 289 300-303.

Skoufias DA, Andreassen PR, Lacroix FB, Wilson L \& Margolis RL 2001 Mammalian mad2 and bub1/bubR1 recognize distinct spindle-attachment and kinetochore-tension checkpoints. PNAS 98 $4492-4497$.

Soewarto D, Schmiady H \& Eichenlaub-Ritter U 1995 Consequences of non-extrusion of the first polar body and control of the sequential segregation of homologues and chromatids in mammalian oocytes. Human Reproduction 10 2350-2360.

Takakura I, Naito K, Iwamori N, Yamashita M, Kume S \& Tojo H 2005 Inhibition of Mitogen Activated Protein Kinase Activity Induces Parthenogenetic Activation and Increases Cyclin B Accumulation during Porcine Oocyte Maturation. Journal of Reproduction and Development.

Taylor S, Scott M \& Holland A 2004 The spindle checkpoint: a quality control mechanism which ensures accurate chromosome segregation. Chromosome Research 12 599-616.

Terret M, Wassmann K, Waizenegger I, Maro B, Peters J \& Verlhac M 2003 The meiosis I-to-meiosis II transition in mouse oocytes requires separase activity. Current Biology 13 1797-1802.

Tsurumi C, Hoffmann S, Geley S, Graeser R \& Polanski Z 2004 The spindle assembly checkpoint is not essential for CSF arrest of mouse oocytes. Journal of Cell Biology 167 1037-1050.

Uhlmann F 2003 Chromosome cohesion and separation: from men and molecules. Current Biology 13 R104-R114.

Verlhac MH, Kubiak JZ, Clarke HJ \& Maro B 1994 Microtubule and chromatin behavior follow MAP kinase activity but not MPF activity during meiosis in mouse oocytes. Development $\mathbf{1 2 0}$ $1017-1025$

Wassmann K, Niault T \& Maro B 2003 Metaphase I arrest upon activation of the MAD2-dependent spindle checkpoint in mouse oocytes. Current Biology 13 1596-1608.

Watanabe Y 2004 Modifying sister chromatid cohesion for meiosis. Journal of Cell Science 117 4017-4023.

Winston NJ 1997 Stability of cyclin B during meiotic maturation and the first meiotic cell division in mouse oocytes. Biologie Cellulaire $89211-219$.

Woods L, Hodges C, Baart E, Baker S, Liskay M \& Hunt P 1999 Chromosomal influence on meiotic spindle assembly: abnormal meiosis I in female Mlh1 mutant mice. Journal of Cell Biology 145 1395-1406.

Yu H, Muszynski MG \& Dawe RK 1999 The maize homologue of the cell cycle checkpoint protein MAD2 reveals kinetochore substructure and contrasting mitotic and meiotic localization patterns. Journal of Cell Biology 145 425-435.

Received 24 June 2005

First decision 24 July 2005

Revised manuscript received 12 September 2005

Accepted 10 October 2005 